\title{
COMPARISON OF ANTHROPOMETRIC PARAMETERS BETWEEN OBESE MALE PARTICIPANTS WITH AND WITHOUT OBSTRUCTIVE SLEEP APNEA
}

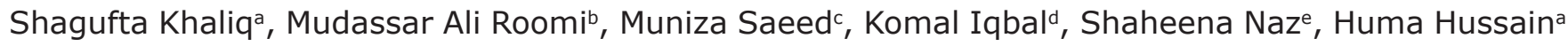 \\ ${ }^{a}$ Assistant Professor, Department of Physiology, Amna Inayat Medical College, Sheikhupura. \\ ${ }^{b}$ Associate Professor, Department of Physiology, Amna Inayat Medical College, Sheikhupura. \\ cProfessor/Head Department of Physiology, Ameer udDin Medical College/PGMI, Lahore. \\ ${ }^{\mathrm{d}}$ Assistant Professor, Department of Physiology, Ameer-ud-Din Medical College/PGMI, Lahore. \\ eAssociate Professor, Department of Physiology, Avicena Medical College, Lahore.
}

\section{ABSTRACT:}

\section{BACKGROUND \& OBJECTIVE:}

The most common risk factors for Obstructive Sleep Apnea includes: Obesity and increased neck circumference in male gender. The objective of the study was to compare the anthropometric parameters between obese male participants with and without obstructive sleep apnea (OSA).

\section{METHODOLOGY:}

Study was conducted at Department of Physiology,Post Graduate Medical Institute, Lahore during 24 August 2014 to 26 May 2015. Obese males ( $n=64$ )with body mass index (BMI) $>25 \mathrm{~kg} / \mathrm{m} 2$ and aged 20-45 years were recruited by convenience sampling. Screening of OSA was made by two subjective tools: STOP BANG Questionnaire, and Berlin Questionnaire while final diagnosis was made by overnight portable pulse oximetry. Study population was divided into two groups. Group-I comprised of 32 obese males with OSA. Group-II had 32 obese males without OSA. BMI, neck circumference, and waist circumference were measured by standard methods. Comparison of variables was done between the groups by Mann-Whitney Utest and t-test.

RESULTS: BMI was higher in group-I than in group-II $(p=0.004)$. Median BMI for group-I was $30.83(28.16-32.80)$ and for group-II was $27.99(26.59-30.08) \mathrm{Kg} / \mathrm{m} 2$. A significantly higher neck circumference $(p<0.001)$ was present in group-I $(41.95 \pm 2.40 \mathrm{~cm})$ than group-II $(39.66 \pm 2.07 \mathrm{~cm})$. Moreover, significantly higher median waist circumference $(p<0.001)$ was present in group-I $(107.5 \mathrm{~cm})$ as compared to group-II $(98.5 \mathrm{~cm})$.

CONCLUSION: Anthropometric parameters (BMI, neck circumference, and waist circumference) are higher in obese individuals with OSA as compared to obese males without OSA.

KEYWORDS: Sleep Apnea Syndromes, Obesity, Waist circumference, Body Mass Index, Neck circumference.

https://doi.org/10.37723/jumdc.v12i3.559

How to cite this:

Khaliq S, Roomi MA, Saeed M, Iqbal K, Naz S, Hussain H. Comparison of anthropometric parameters between obese male participants with and without obstructive sleep apnea. Journal of University Medical \& Dental College. 2021;12(3):162-168.https://doi.org/10.37723/jumdc.v12i3.559

This is an Open Access article distributed under the terms of the Creative Commons Attribution License (http://creativecommons.org/licenses/by/4.0), which permits unrestricted use, distribution, and reproduction in any medium provided the original work is properly cited. 


\section{INTRODUCTION:}

Obstructive sleep apnea (OSA) is a leading health dilemma both in developed and developing countries but its awareness is inadequate. Sleep apnea is a chronic breathing problem that is characterized bya brief cessation of breathing while sleeping due to upper airway obstruction. The repeated and frequent apnea episodes result in sleep disintegration and oxygen desaturation ${ }^{[1]}$. The prevalence of OSA is 3 to $7 \%$ in males and 2 to $5 \%$ in females in both developed and developing countries ${ }^{[1]}$. Obesity is now a worldwide epidemic in both children and adults which poses a serious health burden ${ }^{[2]}$. OSA is aggravated by both central and peripheral adiposity ${ }^{[3]}$. Overweight andobesity are consideredto be reversible risk factors for sleep apnea development and progression. About $40 \%$ of obese people have significant sleep apnea and more than $70 \%$ of patients with sleep apnea have obesity ${ }^{[4]}$.Obesity parameters like abdominal fat, body mass index (BMI), and neck circumference are the major indicators of OSA ${ }^{[4]}$. The neck circumference has been reported to be a powerful clinical predictor of OSA ${ }^{[5,6]}$. However, some researchers have shown that BMI and waist circumference which are indicators of central obesity are better correlated with obstructive sleep apnea than general obesity ${ }^{[7]}$. It has been observed that abdominal obesityis linked with a significant increase in the occurrence of OSA.

Overweight and obesity are considered as the leading reversible risk factors for OSA. Wisconsin Sleep Cohort Study has revealed that $41 \%$ of the adult OSA patients and $58 \%$ of moderateto-severe cases are associated with obesity ${ }^{[1]}$. Untreated sleep apnea may lead to serious health consequences e.g. metabolic syndrome, cardiovascular diseases, lack of concentration, memory problems, psychological depression, daytime sleepiness, increased risk of automobile accidents, and poor quality of life $^{[8]}$. Common features of OSA include excessive daytime sleepiness, witnessed apneas, nocturnal choking, nocturia, tiredness, irritability, non-refreshing sleep, and morning headache ${ }^{[9]}$. A vicious cycle is formed between obesity and OSA due to delays in the diagnosis and management of $\mathrm{OSA}^{[10]}$. Sleep apnea is associated with sleep disturbance, oxidative stress, increased sympathetic activity, systemic inflammation, and glucose intolerance. Moreover, hormonal dysregulation like changes in the blood levels of leptin, ghrelin, and orexin potentially lead to overweight and obesity[10].

Polysomnography is the gold standard test for the diagnosis of obstructive sleep apnea. However, it is complex, expensive, and difficult to access in resource-limited countries such as Pakistan ${ }^{[11]}$. Therefore, it may be very useful for sleep physicians to know the cut-off values of anthropometric measures in their patients of OSA as these measures of obesity can predict the severity of the disease ${ }^{[12,13]}$.

Corresponding Author:

Dr. Shagufta Khaliq

Assistant Professor Department of

Physiology Amna Inayat Medical College,

Sheikhupura.

Email:shaguftakhaliq30@gmail.com

There appears to be a scarcity of clear information on anthropometric measures and the severity of obstructive sleep apnea in Pakistani males. Hence, our objective was to determine and compare the BMI, neck circumference and waist circumference in healthy-looking obese males with and without OSA.

\section{METHODOLOGY:}

It was a cross-sectional study conducted in the Department of Physiology at Post Graduate Medical Institute (PGMI), Lahore, after obtaining ethical approval (No. UHS/ Education/126-131/3320). The duration of this study was 9 months (24 August 2014 to 26 May 2015). We obtained informed consent from each participant after explaining to them thenature and scope of the study. We recruited 64 obese males aged 20-45years having body mass index $>25 \mathrm{~kg} / \mathrm{m} 2$ by convenience sampling. The recruited participants were students and employees of PGMI, Lahore. Median (IQR) of age for group I was 31.00 (28.25-35.00) years and for group-II was $30.50(24.25-32.00)$. There was no statistically significant difference of age between the groups $(p=0.170)$. All the participants with any acute general or upper respiratory tract infection during the last one 
month, chronic inflammatory disease, chronic anxiolytic, and sedatives use, smokers, and alcoholics were excluded from the study.

The screening of OSA was made by two subjective tools: STOP BANG Questionnaire, and Berlin Questionnaire. Participants were divided into high-risk or low-risk categories for obstructive sleep apnea according to the questionnaires. However, the final diagnosis of OSA was made by overnight portable pulse oximetry and those subjects were included in the obstructive sleep apnea group who had $\geq 4 \%$ oxygen desaturation index (ODI) for 5-15 events/hour. Oxygen desaturation index isthe total number of times when oxygen level drops in blood $\geq 4 \%$ from baseline per hour of sleep.

Overnight pulse oximetry was performed by using a portable pulse oximeter (Spirodocpulsox). At bedtime, the pulse oximeter sensor was placed on one fingertip and then the device was attached to the front of the chest. The device was retrieved by the researcher the following morning. The recorded data stored in the memory of the device was observed using a computer via proprietary interface and then it was processed by using appropriate software (Winspiro PRO 5.6.0; Medical International Research).

Depending upon the presence or absence of OSA, the study population was categorized into two groups. Group-I comprised 32 obese male participants having OSA; Group-II included 32 obese male participants without obstructive sleep apnea. Obese male subjects with OSA were further subdivided according to the severity of OSA. Subjects having ODI $>10$ events/hour were included in the group of moderate-to-severe OSA and subjects with ODI 5 to $<10$ events/hour were included in the mild OSA group.

Waist circumference, BMI and neck circumference were recorded by standard anthropometric methods described by WHO. We measured weight in kilograms, height in meters, and neck and waist circumference in centimeters. The weight of the participants was measured in light clothing and bare feet. BMI was calculated as weight $(\mathrm{kg})$ divided by height in square meters (m2). We measured neck circumference, using a flexible measuring tape, at the mid-point of the neck between the superior line of the cricothyroid membrane and mid-cervical spine in a standing position with head upright and eyes facing forward. Waist circumference was measured, over minimal clothing, using a measuring tape at the midpoint between the superior iliac crest and lowest rib. Males having a waist circumference $\geq 90 \mathrm{~cm}$ were considered to have central obesity. The data obtained were entered and analyzed using SPSS version 22. The normality of the data was checked using the Kolmogorov-Smirnov test. Mean \pm standard deviation was used to present normally distributed variables and median (IQR) was given for non-normally distributed variables. Normally distributed quantitative variables were compared by independent samplet-test' and non-normally distributed quantitative variables were compared between the two groups by Mann-Whitney U-test.

\section{RESULTS:}

In group-I, $17(53.12 \%)$ of subjects and $8(25 \%)$ of subjects in group II had BMI $\geq 30 \mathrm{~kg} / \mathrm{m} 2$.BMI of group-I was significantly higher $(p=0.004)$ than the BMI of group-II. Moreover, the neck circumference of group-I was also significantly higher $(p<0.001)$ than group-II (Table I).

Central obesity i.e. waist circumference $\geq 90 \mathrm{~cm}$ was found in $100 \%$ of subjects in group I and $90.62 \%$ of subjects in group-II $(p<0.001)$.

Among the obese male participants with OSA,22 $(69 \%)$ showed moderate to severe OSA and10 $(31 \%)$ had mild OSA. Comparisons of BMI, neck circumference and waist circumference were done between obese participants of mild OSA, moderate-to-severe OSA, and without OSA groups (Table-II). 
Table-I: Comparison of anthropometric parameters between group-I and II.

\begin{tabular}{|c|c|c|c|}
\hline Parameters & $\begin{array}{c}\text { Group-I (n=32) } \\
\text { Obese males with OSA }\end{array}$ & p-value \\
\hline BMI $(\mathrm{kg} / \mathrm{m} 2)^{\mathrm{a}}$ & $30.83(28.16-32.8)^{\mathrm{c}}$ & $27.99(26.59-30.0)^{\mathrm{c}}$ & $0.004^{*}$ \\
\hline $\begin{array}{c}\text { Neck circumference } \\
(\mathrm{cm})^{\mathrm{b}}\end{array}$ & $41.95 \pm 2.4 \mathrm{~d}$ & $39.66 \pm 2.0^{7 \mathrm{~d}}$ & $<0.001^{*}$ \\
\hline $\begin{array}{c}\text { Waist circumference } \\
(\mathrm{cm})^{\mathrm{a}}\end{array}$ & $107.5(102.1-114.0)^{\mathrm{c}}$ & $98.5(93.25-105.25)^{\mathrm{c}}$ & $<0.001^{*}$ \\
\hline
\end{tabular}

aMann Whitney U-test was used for comparison.

bIndependent Sample "t-test" was used for comparison .

"Values are given as median (IQR).

dValues are given as mean \pm SD.

*Statistically significant.

Table-II: Comparison of anthropometric parameters between mild OSA, moderate-tosevere OSA, and without OSA groups.

\begin{tabular}{|c|c|c|c|c|c|c|}
\hline Parameters & $\begin{array}{l}\text { Mild OSA } \\
(n=10)\end{array}$ & $\begin{array}{l}\text { Moderate-to- } \\
\text { Severe OSA } \\
(n=22)\end{array}$ & $\begin{array}{l}\text { Without OSA } \\
\quad(n=32)\end{array}$ & $\begin{array}{c}\text { p-value } \\
\text { (mild OSA vs } \\
\text { moderate-to- } \\
\text { severe OSA) }\end{array}$ & $\begin{array}{c}\text { p-value } \\
\text { (mild OSA } \\
\text { vs without } \\
\text { OSA) }\end{array}$ & $\begin{array}{c}\text { p-value } \\
\text { (moderate-to- } \\
\text { severe OSA vs } \\
\text { without OSA) }\end{array}$ \\
\hline BMI $(\mathrm{kg} / \mathrm{m} 2)$ & $\begin{array}{c}30.09 \\
(27.74-34.73) \\
\end{array}$ & $\begin{array}{c}30.82 \\
(28.32-30.82) \\
\end{array}$ & $\begin{array}{c}27.99 \\
(26.58-30.07)\end{array}$ & 0.684 & 0.095 & $0.006 *$ \\
\hline $\begin{array}{c}\text { Neck } \\
\text { Circumference } \\
(\mathrm{cm})\end{array}$ & $\begin{array}{c}42.0 \\
(41.25-42.32)\end{array}$ & $\begin{array}{c}42.0 \\
(39.72-43.32)\end{array}$ & $\begin{array}{c}39.40 \\
(38.0-41.0)\end{array}$ & 1.000 & $0.008^{*}$ & $0.001 *$ \\
\hline $\begin{array}{l}\text { Waist } \\
\text { Circumference } \\
(\mathrm{cm})\end{array}$ & $\begin{array}{c}107.50 \\
(99.0-110.50)\end{array}$ & $\begin{array}{c}107.50 \\
(102.75-115.37)\end{array}$ & $\begin{array}{c}98.50 \\
(93.25-105.25)\end{array}$ & 0.589 & 0.053 & $<0.001 *$ \\
\hline
\end{tabular}

Mann-Whitney $U$ test was used for comparisons.

Values are given as median (IQR).

*Statistically significant.

\section{DISCUSSION:}

It has been shown that overweight and obesity are reversible risk factors for obstructive sleep apnea. Obesity causes upper airway narrowing which leads to OSA ${ }^{[14]}$. In obese people, the respiratory obstruction may be attributed to narrowing of or opharyngeal passage caused by excess fat along the tonsillar region and soft palate. Moreover, this deposition of fat in the neck is directly associated with an increase in $\mathrm{BMI}^{[15]}$. We observed that both the groups were obese; however, subjects with OSA had significantly greater $\operatorname{BMI}(p=0.004)$.

A bidirectional association has been observed between OSA and obesity. Obesity is a traditional risk factor for OSA, which itself leads to more obesity in a variety of ways like daytime somnolence, decrease in physical activity, and hormonal dysregulation ${ }^{[4,16]}$. Our study also analyzed different anthropometric measures in various severity levels of OSA. The OSA group is divided into mild OSA (ODI 5 to $<10$ ) and moderate-to-severe OSA (ODI $>10)^{[17]}$. We have observed that comparison of moderate to severe OSA and without OSA groups had statistically significant difference $(p=0.006)$ of BMI.

Neck circumference is a simple and time-saving indicator of upper body obesity ${ }^{[18]}$. Several studies have suggested that a thick neck is an important and effective factor in determining OSA. Neck circumference is usually greater among men and it is a newly recognized medical aspect that may 
be related to OSA. In our study, a statistically significant difference in neck circumference has been found between the obese participants with and without OSA $(p<0.001)$. Additionally, we found that individuals even with mild OSA have a median neck circumference of $42 \mathrm{~cm}$ or higher. Some studies have shown that he neck circumference of $40 \mathrm{~cm}$ or higher has greater sensitivity and specificity in predicting OSA than BMI regardless of gender ${ }^{[19]}$.

In the study significant difference in neck circumference is found when OSA is subdivided according to the severity level of OSA and compared with obese participants without OSA. In our study, the comparison of the without OSA group with the mild OSA group showed a significant $(p=0.008)$ difference. Moreover, when we compared without OSA group with the moderate to severe OSA group, a significant difference $(p=0.001)$ was observed. There was no significant difference between mild OSA group and the moderate to severe OSA group. However, in another study, it has been reported that neck circumference in severe OSA cases was significantly higher than in non-severe OSA patients and the same research also concluded that neck circumference is an independent risk factor for severe OSA ${ }^{[20]}$. The probable scientific reason for this variation of result of present study from given study may be that they used apnea hypopnea index (AHI) to evaluate the severity of OSA. AHI $\geq 30$ was described as severe OSA and $\mathrm{AHI}<30$ was described as non-severe(mild to moderate OSA). However, we use oxygen desaturation index to describe the severity of OSA.

Waist circumference, indicative of central obesity, is also considerably different between the two study groups $(p<0.001)$, values being higher in obese participants with OSA. Our results are similar to a study that has concluded that there is an independent relationship of OSA with obesity among young adults ${ }^{[7,8]}$. Central obesity promotes soft tissue enlargement around the airways leading to narrowing of the pharyngeal airways. The decreased lung volume increases pharyngeal wall collapsibility by decreasing longitudinal tracheal traction. This lung volume hypothesis suggests that waist circumference may be a superiorindicator for OSA than body mass indexor neck circumference ${ }^{[21]}$.
The risk of OSA increases by six-folds with a 10 $\%$ increase in body weight. Moreover, a $10 \%$ reduction in weight leads to a-26 \% decrease in oxygen desaturation ${ }^{[22]}$.In another study it is also assessd that increase BMI and neck circumference also increases the risk of OSA ${ }^{[23]}$. Furthermore, obesity is also common in the patients with OSA. A dipocytes, especially in the visceral fat, secrete cytokines that lead to sterile inflammation observed in obese people ${ }^{[22]}$. Therefore reduction in body weight may improve the risk of development of OSA.

\section{CONCLUSION:}

There was a significant difference in BMI between the two groups even though all of our study subjects were obese according to the South Asian criteria for obesity. The BMI, neck circumference and waist circumference were significantly higher in obese males with OSA than obese males without OSA.We also found that the BMI, neck circumference and waist circumference were significantly higher in the moderate to severe OSA group than in subjects without OSA.

Lifestyle modifications (eating behaviors, smoking, drinking alcohol, etc.) and increase in physical activity are some of the strategies to decrease overweight and obesity. By adopting these life style modifications, the consequences of obesity, OSA and risk of cardiovascular diseases will be greatly reduced. It is need of the hour to create public awareness about the importance of weight loss by lifestyle modifications.

\section{ACKNOWLEDGEMENT:}

We are greatly thankful to Dr. Muhammad Bin Naseer for his assistance in recording the anthropometric parameters of the study participants.

CONFLICT OF INTEREST: None. GRANT SUPPORT \& FINANCIAL DISCLOSURE: None. 


\section{REFERENCES:}

1. Punjabi NM. The epidemiology of adult obstructive sleep apnea. Proceedings of the American Thoracic Society. 2008; 5(2):136143.

2. Bellew W, Bauman A, Kite J, Foley B, Reece $L$, Thomas $M$, et al. Obesity prevention in children and young people; what policy actions are needed. Public Health Research and Practice. 2019;29(1):e2911902. Doi: 10.17061/phrp2911902

3. Almendros I, Martinez-Garcia MA, Farré R, Gozal D. Obesity, sleep apnea, and cancer. International Journal of Obesity. 2020;44(8):1653-1667.

4. Carter R, Watenpaugh DE. Obesity and obstructive sleep apnea: Or is it OSA and obesity? Pathophysiology. 2008;15:71-77. Doi: 10.1016/j.pathophys.2008.04.009

5. Edmonds PJ, Gunasekaran K, Edmonds LC. Neck grasp predicts obstructive sleep apnea in type 2 diabetes mellitus. Sleep Disorders. 2019.Doi: $10.1155 / 2019 / 3184382$

6. Zhang L, Zeng T, Gui Y, Sun Y, Xie F, Zhang $D$, et al. Application of neck circumference in four-variable screening tool for early prediction of obstructive sleep apnea in acute ischemic stroke patients. Journal of Stroke and Cerebrovascular Diseases. 2019;28(9):2517-2524. Doi: 10.1016/j. jstrokecerebrovasdis.2019.06.011

7. Zhao X, Xu H, Qian Y, Liu Y, Zou J, Yi H, et al. Abdominal obesity is more strongly correlated with obstructive sleep apnea than general obesity in China: results from two separated observational and longitudinal studies. Obesity Surgery. 2019;29(8):25352547.

8. Pillar G, Shehadeh N. Abdominal fat and sleep apnea: the chicken or the egg?. Diabetes Care. 2008;31(2):303-309. Doi:10.2337/ dc08-s272

9. Knauert M, Naik S, Gillespie MB, Kryger M. Clinical consequences and economic costs of untreated obstructive sleep apnea syndrome. World Journal of Otorhinolaryngology - Head \& Neck Surgery. 2015;1:17-27. Doi:10.1016/j. wjorl.2015.08.001
10. Stansbury RC, Strollo PJ. Clinical manifestations of sleep apnea. Journal of Thoracic Disease. 2015;7(9):298-310. Doi: $10.3978 / j$.issn.2072-1439.2015.09.13

11. Liu WT, Wu HT, Juang JN, Wisniewski A, Lee $H C$, Wu $D$, et al. Prediction of the severity of obstructive sleep apnea by anthropometric features via support vector machine. PLoSOne. 2017;12(5):e0176991. Doi:10.1371/journal.pone.0176991

12. Remya KJ, Mathangi K, Mathangi DC, Sriteja Y, Srihari R, Govindaraju S, et al. Predictive value of craniofacial and anthropometric measures in obstructive sleep apnea (OSA). The Journal of Craniomandibular \& Sleep Practice. 2017;35(3):162-167. Doi:10.1080 /08869634.2016.1206701

13. Soylu AC, Levent E, Sarıman N, Yurtlu Ş, Alparslan S, Saygı A. Obstructive sleep apnea syndrome and anthropometric obesity indexes. Sleep and Breathing. 2012;16(4):1151-1158.

14. Busetto L, Enzi G, Inelmen EM, Costa G, Negrin V, Sergi G, et al. Obstructive sleep apnea syndrome in morbid obesity: effects of intragastric balloon. Chest. 2005;128(2):618623. Doi:10.1378/chest.128.2.618.

15. Modena DA, Cazzo E, Cândido EC, Baltieri L, Silveira LJ, Almeida AM, et al. Obstructive sleep apnea syndrome among obese individuals: A cross-sectional study. Revista Da Associacao Medica Brasileira. 2017;63(10):862-868. Doi:10.1590/1806-9282.63.10.862

16. Hamilton GS, Joosten SA. Obstructive sleep apnoea and obesity. Australian Family Physician. 2017;46(7):460-463.

17. Chung $F$, Liao $P$, Elsaid H, Islam S, Shapiro $\mathrm{CM}$, Sun Y. Oxygen desaturation index from nocturnal oximetry: a sensitive and specific tool to detect sleep-disordered breathing in surgical patients. Anesthesia \& Analgesia. 2012;114(5):993-1000.

18. Koppad AK, Kaulgud RS, Arun BS. A study of correlation of neck circumference with Framingham risk score as a predictor of coronary artery disease. Journal of clinical and diagnostic research. Journal of Clinical and Diagnostic Research.2017;11(9): OC17-OC20.Doi: 10.7860/JCDR/2017/25710.10609 
19. Ho ML, Brass SD. Obstructive sleep apnea. Neurology International. 2011; 3: 60-67. Doi:10.4081/ni.2011.e15

20. Ahbab S, Ataoğlu HE, Tuna M, Karasulu L, Çetin F, Temiz LÜ, et al. Neck circumference, metabolic syndrome and obstructive sleep apnea syndrome; evaluation of possible linkage. Medical science monitor. International Medical Journal of Experimental and Clinical Research. 2013;19:111-117.Doi: 10.12659/ MSM. 883776

21. Isono S. Obstructive sleep apnea of obese adults pathophysiology and perioperative airway management. Anesthesiology. 2009;110: 908-921. Doi:10.1097/ ALN.0b013e31819c74be

22. Bauters F, Rietzschel ER, Hertegonne $\mathrm{KB}$ and Chirinos JA. The Link Between Obstructive Sleep Apnea and Cardiovascular Disease. Current Atherosclerosis Reports. 2016;18(1):1-11. Doi: 10.1007/s11883015-0556-z.

23. Ullah MU, Ansar JK, Mustafavi S, Iftikhar R, Raja W, Shah WH, et al. The effect of adding body mass index (BMI) and neck circumference to Epworth sleepiness scale (ESS) for diagnosing obstructive sleep apnea hypopnea syndrome in sleep clinics. Pakistan Armed Forces Medical Journal. 2016;66(1):57-61.

\section{Author's Contribution:}

Shagufta Khaliq: Conception and design, acquisition of data, analysis and interpretation of data, drafting the article.

Mudassar Ali Roomi: Analysis and interpretation of data, Drafting the article, critical review of article.

Muniza Saeed: Conception and design, interpretation of data, critical review of article

Komal Iqbal: Data collection, manuscript writing, critical review of article.

Shaheena Naz: Statistical analysis, manuscript writing and critical review of article.

Huma Hussain: Manuscript writing and critical review of article.

Submitted for Publication: 15-01-2021

Accepted After revision: 06-07-2021 